TITLE:

\title{
Numerical study on effective configurations of the Knudsen pump for separation and compression
}

\author{
$\operatorname{AUTHOR}(\mathrm{S})$ : \\ Takata, Shigeru; Umetsu, Hiroki
}

\section{CITATION:}

Takata, Shigeru ...[et al]. Numerical study on effective configurations of the Knudsen pump for separation and compression. AlP Conference Proceedings 2011, 1333: 998-1003

\author{
ISSUE DATE: \\ 2011 \\ URL: \\ http://hdl.handle.net/2433/160667 \\ RIGHT: \\ Copyright 2011 American Institute of Physics. This article may be downloaded for \\ personal use only. Any other use requires prior permission of the author and the \\ American Institute of Physics. The following article appeared in AIP Conference \\ Proceedings 1333, 998 (2011) and may be found at http://link.aip.org/link/?apc/1333/998
}




\title{
Numerical study on effective configurations of the Knudsen pump for separation and compression
}

\author{
Shigeru Takata ${ }^{\dagger} \ddagger$ and Hiroki Umetsu ${ }^{\dagger}$ \\ $\dagger$ Department of Mechanical Engineering and Science, Kyoto University, Kyoto 606-8501, Japan \\ $\$$ Advance research Institute of Fluid Science and Engineering, Kyoto University, Kyoto 606-8501, Japan
}

\begin{abstract}
Performance of the Knudsen pump is studied numerically to find out effective configurations not only for the finally achieved separation and compression rates, but also for its time efficiency. A system of convection-diffusion equations with transmission conditions, which have been derived from the Boltzmann equation under the narrow channel approximation, is used for simulations of various configurations. A comparison with the experiment of evacuation from a tank is also made to demonstrate the practical ability of that system.
\end{abstract}

Keywords: Knudsen pump, gas separation, Boltzmann equation, narrow channel approximation, thermal transpiration, microfluidics PACS: 47.45.-n, 51.10.+y, 05.20.Dd, 47.61.-k, 47.15.G-

\section{INTRODUCTION}

Knudsen pump is a non-mechanical pumping system that makes use of the thermal transpiration. A prototype is a cascade of elemental units composed of thin and relatively thick channels with increasing and decreasing wall temperature along the channel (Fig. 1) [1, 2, 3]. We have recently pointed out that the pump can also be used as a gas separator [4]. In the present paper, we will report some results on effective channel configurations both in separation and compression rates and in time performance within the framework of narrow channel approximation with transmission conditions [4]. This framework that basically

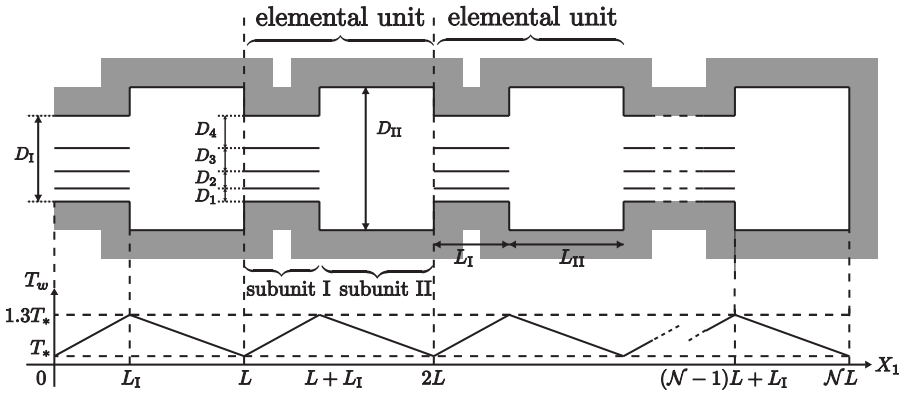

FIGURE 1. Knudsen pump originated from Aoki \& Degond [5] enabled us to derive a system of (macroscopic) convection-diffusion equations with transmission condition systematically from the Boltzmann equation. We will show the results obtained by a large number of simulations by the use of this system. The corresponding simulations by other usual direct methods are too time-consuming to be performed. We also show that the data of evacuation experiment by Sone \& Sugimoto [6] are recovered reasonably well by our approach.

\section{PROBLEM AND BASIC EQUATIONS}

Let us consider the Knudsen pump composed of $\mathscr{N}$ elemental units with length $L$ (Fig. 1). Each of them is further composed of subunits I and II: the former is divided into $N$ parallel thin channels with length $L_{\mathrm{I}}$ and width $D_{i}$ $\left(i=1,2, \ldots, N ; D_{\mathrm{I}}=D_{1}+\cdots+D_{N}\right)$, while the latter is one relatively thick channel with length $L_{\mathrm{II}}\left(L=L_{\mathrm{I}}+L_{\mathrm{II}}\right)$ and width $D_{\mathrm{II}}$. The wall temperature is saw-like periodic in harmony with the cascade structure. We investigate the time-dependent behavior of a binary gas mixture of species A and B in the pump by the use of the convection-diffusion set of equations which has been derived in Ref. [4] from the Boltzmann equation by a systematic asymptotic analysis for small aspect ratio $\varepsilon$ [ $\varepsilon=\max \left(D_{\mathrm{I}} / L_{\mathrm{I}}, D_{\mathrm{II}} / L_{\mathrm{II}}\right) \ll 1$; narrow channel approximation]. In the actual computations, we make use of the set equipped with numerical table of transport coefficients based on the McCormack model equation [7] and diffuse reflection boundary condition. This table has been prepared in Ref. [8] for several intermolecular 
potentials and combinations of gas species.

Let us introduce the dimensionless quantities corresponding to the position $X_{1}$, time $t$, number density $n^{\alpha}$ of species $\alpha(\alpha=\mathrm{A}, \mathrm{B})$, number density $n$, pressure $p$, and temperature $T$ of total mixture, and temperature $T_{\mathrm{w}}$ of channel walls:

$$
x=X_{1} / L, \quad \hat{t}=t / t_{*}, \quad \hat{n}^{\alpha}=n^{\alpha} / n_{*}, \quad \hat{n}=n / n_{*}, \quad \hat{p}=p / p_{*}, \quad \hat{T}=T / T_{*}, \quad \hat{T}_{\mathrm{w}}=T_{\mathrm{w}} / T_{*},
$$

where $L, t_{*}, n_{*}, p_{*}$, and $T_{*}$ are the reference length (in $X_{1}$ direction), time, number density, pressure, and temperature. Here $t_{*}$ and $p_{*}$ are chosen in such a way that $t_{*}=L^{2} D_{\mathrm{I}}^{-1}\left(2 k T_{*} / m^{\mathrm{A}}\right)^{-1 / 2}$ and $p_{*}=n_{*} k T_{*}$ with $m^{\mathrm{A}}$ and $k$ being the mass of a molecule and Boltzmann's constant respectively. Note that the concentration $\chi^{\alpha}$ of species $\alpha$ is written by $\chi^{\alpha}=\hat{n}^{\alpha} / \hat{n}$ and that the relations $\hat{p}=\hat{n} \hat{T}$ and $\hat{n}=\hat{n}^{\mathrm{A}}+\hat{n}^{\mathrm{B}}$ hold.

When $\varepsilon$ is small enough, $\hat{T}=\hat{T}_{\mathrm{W}}$ and the (dimensionless) number densities $\hat{n}^{\alpha}$ are uniform in each cross-section of the channels within the error of $O(\varepsilon)$, i.e., $\hat{T}$ and $\hat{n}^{\alpha}$ depend only on $\hat{t}$ and $x$ in each channel up to $O\left(\varepsilon^{0}\right)$. Furthermore, the behavior of $\hat{n}^{\alpha}$ up to $O\left(\varepsilon^{0}\right)$ is described by the following set of convection-diffusion equations with the connection conditions at junctions [4].

$\underline{\left.\text { In the } i \text {-th channel of subunit I [for } j<x<j+\left(L_{\mathrm{I}} / L\right)\right]}$ :

$$
0=\partial_{t} \hat{n}_{i}^{\alpha}+\partial_{x} \mathscr{J}_{i}^{\alpha}, \quad \mathscr{J}_{i}^{\alpha}=\left(D_{i} / D_{\mathrm{I}}\right)\left(M_{\mathrm{P}}^{\alpha} \partial_{x} \ln \hat{p}_{i}+M_{\mathrm{T}}^{\alpha} \partial_{x} \ln \hat{T}_{\mathrm{w}}+M_{\chi}^{\alpha} \partial_{x} \chi_{i}^{\mathrm{A}}\right)\left(\hat{p}_{i} / \hat{T}_{\mathrm{w}}^{1 / 2}\right),
$$

In the channel of subunit II [for $j+\left(L_{\mathrm{I}} / L\right)<x<j+1$ ]:

$$
0=\partial_{t} \hat{n}_{\mathrm{II}}^{\alpha}+\partial_{x} \mathscr{J}_{\mathrm{II}}^{\alpha}, \quad \mathscr{J}_{\mathrm{II}}^{\alpha}=\left(D_{\mathrm{II}} / D_{\mathrm{I}}\right)\left(M_{\mathrm{P}}^{\alpha} \partial_{x} \ln \hat{p}_{\mathrm{II}}+M_{\mathrm{T}}^{\alpha} \partial_{x} \ln \hat{T}_{\mathrm{W}}+M_{\chi}^{\alpha} \partial_{x} \chi_{\mathrm{II}}^{\mathrm{A}}\right)\left(\hat{p}_{\mathrm{II}} / \hat{T}_{\mathrm{W}}^{1 / 2}\right),
$$

At junctions [for $x=1,2, \ldots, \mathscr{N}-1$ and $\left.x=j+\left(L_{\mathrm{I}} / L\right)\right]$ :

$$
\hat{n}_{i}^{\alpha}=\hat{n}_{\mathrm{II}}^{\alpha}, \quad \sum_{l=1}^{N}\left(D_{l} / D_{\mathrm{I}}\right) \mathscr{J}_{l}^{\alpha}=\left(D_{\mathrm{II}} / D_{\mathrm{I}}\right) \mathscr{J}_{\mathrm{II}}^{\alpha}
$$

where $i=1,2, \ldots, N$ and $j=0,1, \ldots, \mathscr{N}-1$. In the above equations, we have added the subscripted $i$ and II to the unknown quantities in order to indicate the corresponding channel. $M_{J}^{\alpha}(J=\mathrm{P}, \mathrm{T}, \chi)$ occurring in the above equations are functions of $\hat{n}^{\alpha}$ (and $\hat{T}_{\mathrm{w}}$ ) that take the following form in the channel $q(q=1,2, \ldots, N$, II)

$$
M_{J}^{\alpha}=M_{J}^{\alpha}\left(\mathrm{K}_{q}, \chi_{q}^{\mathrm{A}}, \hat{T}_{\mathrm{w}}\right), \quad \mathrm{K}_{q}=\left(D_{\mathrm{I}} / D_{q}\right)\left(\hat{T}_{\mathrm{w}} / \hat{p}_{q}\right) \mathrm{K}_{*},
$$

where $\mathrm{K}_{*}$ is the reference Knudsen number defined by $\ell_{*} / D_{\mathrm{I}}$ with $\ell_{*}$ being the mean free path of a molecule in the pure gas A in the equilibrium state at rest with number density $n_{*}$ and temperature $T_{*}$. Thus, the equations (1) and (2) are a nonlinear convection-diffusion equation. $\mathrm{K}_{q}$ and $\mathscr{J}_{q}$ represent the local Knudsen number and the particle flux through the channel $q$, while $M_{J}^{\alpha}$ the particle fluxes in elementary channel flow problems described by the linearized Boltzmann (or its model) equation. In the present study, we will make use of the numerical table of $M_{J}^{\alpha}$ prepared in Ref. [8] on the basis of the McCormack model for Lennard-Jones 12-6 molecules and diffuse reflection condition.

We will study the situation where the extreme left end $(x=0)$ is open, the extreme right end $(x=\mathscr{N})$ is closed, and the saw-like temperature distribution is given by

$$
\hat{T}_{\mathrm{w}}(x)=\left\{\begin{array}{ll}
1+0.6|x-j| & j<x<j+\left(L_{\mathrm{I}} / L\right), \\
1+0.6|x-j-1| & j+\left(L_{\mathrm{I}} / L\right)<x<j+1,
\end{array} \quad(j=0,1, \ldots, \mathscr{N}-1) .\right.
$$

Actually, we solve the system (1)-(3) numerically with the following boundary and initial conditions

$$
\begin{aligned}
& \hat{n}_{i}^{\mathrm{A}}(t, x=0)=c^{\mathrm{A}}, \quad \hat{n}_{i}^{\mathrm{B}}(t, x=0)=1-c^{\mathrm{A}} \quad(i=1,2, \ldots, N), \\
& \mathscr{J}_{\mathrm{II}}^{\mathrm{A}}(t, x=\mathscr{N})=\mathscr{J}_{\mathrm{II}}^{\mathrm{B}}(t, x=\mathscr{N})=0, \\
& \hat{n}_{q}^{\mathrm{A}}(t=0, x)=c^{\mathrm{A}}, \quad \hat{n}_{q}^{\mathrm{B}}(t=0, x)=1-c^{\mathrm{A}} \quad(q=1,2, \ldots, N, \mathrm{II}),
\end{aligned}
$$

where $c^{\mathrm{A}}$ is a constant such that $0 \leq c^{\mathrm{A}} \leq 1$. This setting implies that the lowest temperature of the wall and the constant number density of the gas outside of the open end are chosen as the reference temperature $T_{*}$ and number density $n_{*}$. Rigorously speaking, the open end is not treated faithfully, i.e., the immediate outside of it is assumed to 


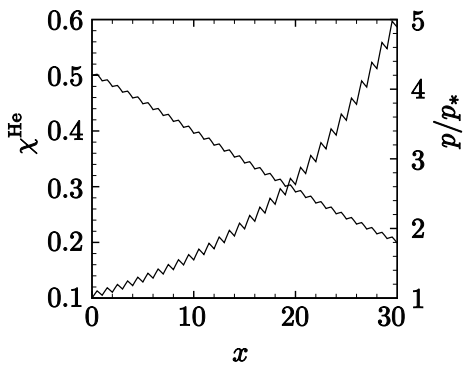

FIGURE 2. Concentration and pressure profiles at the final steady state for a He-Ar mixture (A:

Helium, B: Argon) in the case $N=20$ and $\mathscr{N}=30$.

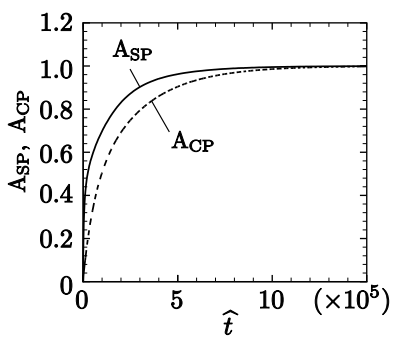

(a)

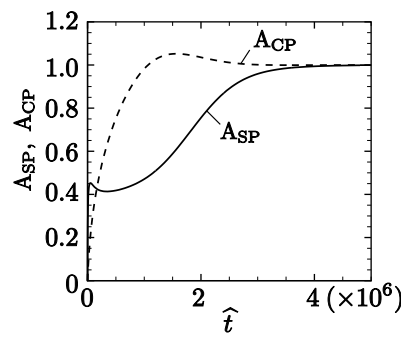

(b)
FIGURE 3. Time evolution of achievement rates for a He-Ar mixture (A: Helium, B: Argon). (a) The case of $N=10$ and $\mathscr{N}=100$, (b) that of $N=5$ and $\mathscr{N}=300$.

be regulated at a constant resting state. On the other hand, the condition at the closed end implies no net flow through the end, which is reasonable. The initial condition implies that the gas in the cascade system is initially at the same state as the gas outside of the open end.

\section{NUMERICAL COMPUTATIONS AND RESULTS}

\section{Simulation set up and measure for performance evaluation}

There are many parameters in the system (1)-(8): $\mathrm{K}_{*}, c^{\mathrm{A}}, \mathscr{N}, N, L_{\mathrm{I}} / L, D_{\mathrm{II}} / D_{\mathrm{I}}$, and $D_{i} / D_{\mathrm{I}}$, where $i=1, \ldots, N$. In what follows, unless otherwise stated, the parameters other than $\mathscr{N}$ and $N$ are set to be the following values:

$$
\mathrm{K}_{*}=1, \quad c^{\mathrm{A}}=1 / 2, \quad L_{\mathrm{I}} / L=1 / 2, \quad D_{\mathrm{II}} / D_{\mathrm{I}}=1, \quad D_{i} / D_{\mathrm{I}}=1 / N \quad(i=1, \ldots, N) .
$$

This setting is motivated by the features at final steady states [4] that (i) the pump is most effective at intermediate reference Knudsen number, (ii) $L_{\mathrm{I}} / L$ does not influence both in separation and compression, and (iii) the configuration with $D_{\mathrm{II}} / D_{\mathrm{I}}=1$ and $D_{i} / D_{\mathrm{I}}=1 / N$ for $\mathrm{K}_{*}=1$ is identical to that with $D_{\mathrm{II}} / D_{\mathrm{I}}=N$ and no subdivision for $K_{*}=N$.

Figure 2 shows typical concentration and pressure profiles at the final steady state. Except for the saw-like behavior, they change monotonically in $x$. Keeping in mind this feature, we introduce the measures of performance based on the quantities at the closed end:

$$
\begin{array}{ll}
\mathrm{SP}=\frac{c^{\mathrm{A}}-\chi^{\mathrm{A}}(\hat{t}=\infty, x=\mathscr{N})}{c^{\mathrm{A}}}, & \mathrm{A}_{\mathrm{SP}}(\hat{t})=\frac{\left|c^{\mathrm{A}}-\chi^{\mathrm{A}}(\hat{t}, x=\mathscr{N})\right|}{\mid c^{\mathrm{A}}-\chi^{\mathrm{A}(\hat{t}=\infty, x=\mathscr{N}) \mid},} \\
\mathrm{CP}=\hat{p}(\hat{t}=\infty, x=\mathscr{N})-1, & \mathrm{~A}_{\mathrm{CP}}(\hat{t})=\frac{|\hat{p}(\hat{t}, x=\mathscr{N})-1|}{|\hat{p}(\hat{t}=\infty, x=\mathscr{N})-1|},
\end{array}
$$

with $c^{\mathrm{A}}=1 / 2$. Here SP and CP respectively represent the degrees of separation and compression at the final steady state, while $\mathrm{A}_{\mathrm{SP}}$ and $\mathrm{A}_{\mathrm{CP}}$ represent the achievement rate at time $\hat{t}$ relative to SP and CP. The positive SP and CP respectively imply that the species $\mathrm{B}$ is enriched and the mixture is compressed at the closed end. Most typically, $\mathrm{A}_{\mathrm{SP}}$ and $A_{C P}$ increase monotonically in $\hat{t}$ from zero to unity as in Fig. 3(a). However, in some cases, they reach a local maximum as in Fig. 3(b). In particular, $A_{C P}$ may exceeds unity slightly. We introduce the times $\hat{t}_{\mathrm{SP}}$ and $\hat{t}_{\mathrm{CP}}$ at which $\mathrm{A}_{\mathrm{SP}}$ and $\mathrm{A}_{\mathrm{CP}}$ reach 0.99 for the first time:

$$
\hat{t}_{\mathrm{SP}}=\min \left\{\hat{t} \mid \mathrm{A}_{\mathrm{SP}}(\hat{t})=0.99\right\}, \quad \hat{t}_{\mathrm{CP}}=\min \left\{\hat{t} \mid \mathrm{A}_{\mathrm{CP}}(\hat{t})=0.99\right\} .
$$

We use them as the measure of time performance, together with the degrees of separation SP and compression CP.

\section{Main results}

All the results shown in this section are for a Helium-Argon mixture (A: Helium, B: Argon). For other mixtures of rare gases such as Helium-Neon, Neon-Argon, the corresponding simulations are performed for $\mathrm{K}_{*}=1$ and the results are qualitatively the same. 


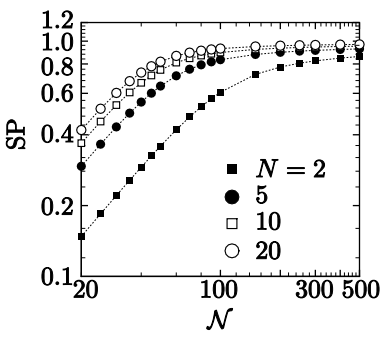

(a)

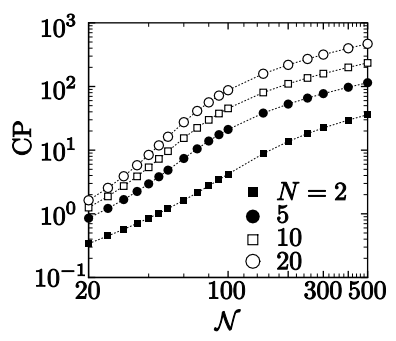

(b)

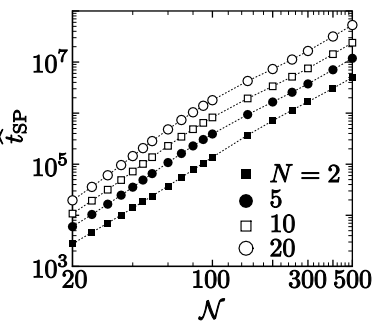

(a)

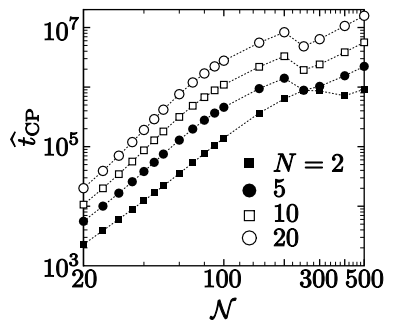

(b)

FIGURE 4. Separation and compression performances SP and $\mathrm{CP}$ as a function of the number of unit $\mathscr{N}$ for $N=2,5,10$, and 20. (a) SP vs. $\mathscr{N}$, (b) CP vs. $\mathscr{N}$.

FIGURE 5. Consumed times $\hat{t}_{\mathrm{SP}}$ and $\hat{t}_{\mathrm{CP}}$ as a function of the number of unit $\mathscr{N}$ for $N=2,5,10$, and 20. (a) $\hat{t}_{\text {SP }}$ vs. $\mathscr{N}$, (b) $\hat{t}_{\text {CP }}$ vs. $\mathscr{N}$.

Figure 4 shows the dependence of degrees of separation and compression (SP and CP) on the number of cascade $\mathscr{N}$. The results for different numbers of division $N$ of subunit I are shown in the figure. As was reported in [4], both SP and CP increase as $N$ and $\mathscr{N}$ increase. The dotted lines in the figure, which connect the data for the same $N$, are the same, except for the saw-like part, as the profiles $1-\chi^{\mathrm{A}} / c^{\mathrm{A}}$ and $\hat{p}-1$ at the final steady state in $20 \leq x \leq 500$ of the cascade system of $\mathscr{N}=500$. This means that it is the elemental units near the closed end that are responsible for the improvement of SP and CP when $\mathscr{N}$ increased. Thus, the saturation of SP for large $\mathscr{N}$ implies that those units do not function, which can be understood by the high CP value there. That is, SP tends to saturate when CP reaches around $10^{2}$, implying the effective Knudsen number near the closed end is around 0.01 and resulting in the loss of rarefaction effect. By comparison between panels (a) and (b), the function as a separator is seen to be much less effective than that as a compressor in low Knudsen number regime.

Figure 5 shows the consumed times $\hat{t}_{\mathrm{SP}}$ and $\hat{t}_{\mathrm{CP}}$ required to achieve the $99 \%$ of SP and CP. As seen in the figure, increasing $N$ and $\mathscr{N}$ worsens the time performance as a whole, in contrast to that it has improved the degrees of separation and compression (Fig. 4). $\hat{t}_{\mathrm{CP}}$ changes unnaturally in $\mathscr{N}$ around $\mathscr{N}=200$, which is simply due to the occurrence of the non-monotonic behavior of $\mathrm{A}_{\mathrm{CP}}$ (see the dashed line in Fig. 3). If we lower the criterion of consumed time from $99 \%$ to some lower value, the odd behavior does not occur.

In summary, the increase of $N$ and $\mathscr{N}$ improves the degrees of separation and compression, but the time efficiency becomes worse. Thus, we have to trade off the degrees of separation and compression against the time efficiency. Plotting SP versus $\hat{t}_{\mathrm{SP}}$ and $\mathrm{CP}$ versus $\hat{t}_{\mathrm{CP}}$, which is shown in Fig. 6, serves as a guideline for finding optimal configurations. In such a plotting, the result for the configuration efficient both in separation (or compression) and in time tends to appear in the upper left of the figure. Thus, for instance, the construction of cascade is more effective by using the units with $N=5 \sim 10$ than with $N=2$. In order to achieve the same degree of separation, the cascade of small number of units with $N=5 \sim$

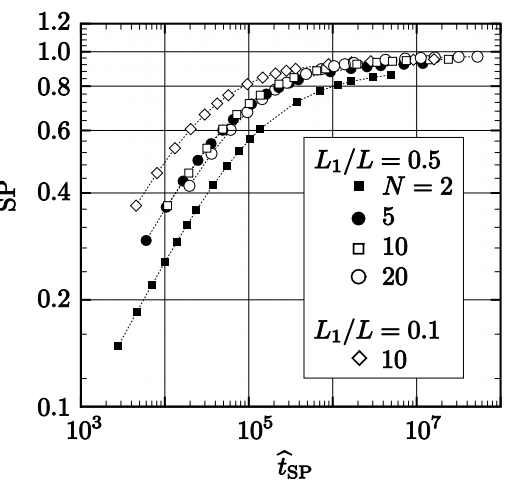

(a)

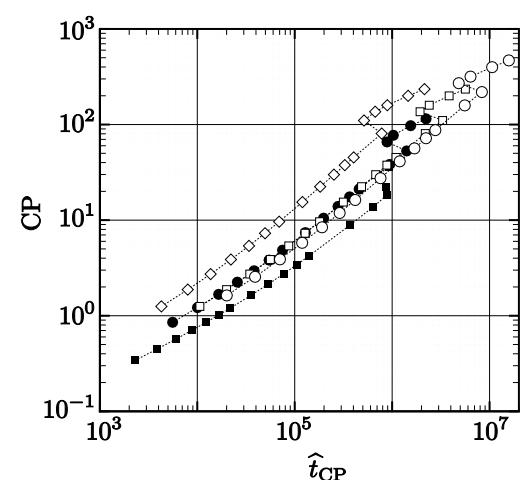

(b)
FIGURE 6 Separation and compression performance versus consumed time for 99\% achievement. (a) Separation performance SP vs. $\hat{t}_{\mathrm{SP}}$, (b) compression performance $\mathrm{CP}$ vs. $\hat{t}_{\mathrm{CP}}$. In both panels, along each dotted line, symbols indicate the results, from left to right, for $\mathscr{N}=20,25,30,35,40,45,50,60,70,80,90,100,150$, 200, 250, 300, 400, and 500 .

10 is superior in time performance to that of large number of units with $N=2$. The cascade of units with much larger $N$ requires less number of units but in place becomes time consuming, though it can achieve a higher separation rate.

When $\mathrm{K}_{*}=0.1$, the optimal division is raised to $N \sim 20$, while it remains $N=5 \sim 10$ when $\mathrm{K}_{*}=10$. However, since the pump will be designed to perform best at $\mathrm{K}_{*} \sim 1$, the above difference would not seriously be considered.

If the part of subunit $\mathrm{I}$ is shortened, the time performance is found to be improved, which is in remarkable contrast to no influence on SP and CP. In Fig. 6, the data for $L_{\mathrm{I}} / L=0.1$ and $N=10$ are also shown for comparison. Our simulation results for other values of $L_{\mathrm{I}} / L$ showed that the smaller $L_{\mathrm{I}} / L$, the better the performance in time. However, there should 


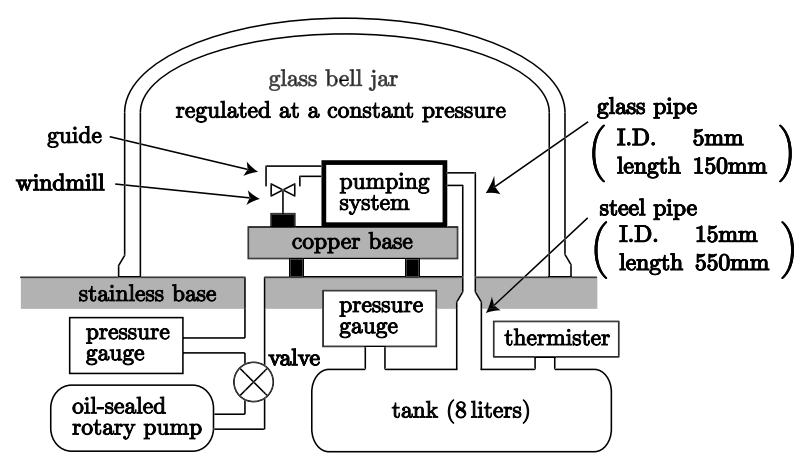

(a)

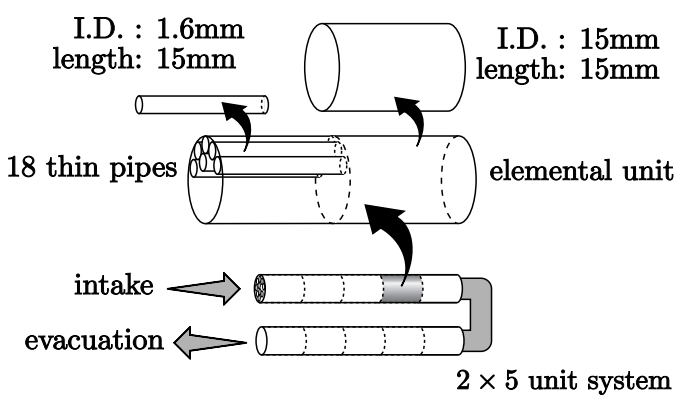

(b)

FIGURE 7. Sketch of experiment in [6]. (a) Whole system (by courtesy of H. Sugimoto), (b) details of the pumping system.

be an optimal value of $L_{\mathrm{I}} / L$ for a given length of elemental unit $L$. The discrepancy is due to the narrow channel approximation because the optimization within this constraint violates the assumption required for the approximation when $L_{\mathrm{I}} / L$ is close to 0 or 1 . A study by some other method is in order for this optimization problem.

Incidentally, in the case of $L_{\mathrm{I}} / L=0.1, N=10 \sim 20$ was found to be optimal. Judging from the data for various $L_{\mathrm{I}} / L$, the subdivision of subunit I into around 10 (i.e., $N \sim 10$ ) would be a nearly optimal configuration of the elemental unit.

\section{SUPPLEMENTAL TEST OF RELIABILITY OF THE PRESENT APPROACH}

Main advantage of our approach, the use of convection-diffusion system (1)-(3), is its simplicity due to the contraction of variables, which dramatically reduces computational costs. We stress that it is too time consuming to obtain the corresponding results in the previous section by a usual method like DSMC. Since the system (1)-(3) has been derived from the Boltzmann equation, our approach has a firm theoretical basis. In fact, comparisons made so far on the final steady state agreed well with computational results by DSMC or other kinetic equations (e.g., [4, 9]). We will show below how well our approach predicts real experimental results, aiming at the demonstration of its practical usefulness. The experiment to be compared with is on the evacuation from a tank done by Sone \& Sugimoto [6].

Figure 7 is a sketch of the experiment. The pump is composed of two subsystems with 5 elemental units. The subsystems are connected by a curved glass pipe. Each elemental unit is a straight glass pipe (length $30 \mathrm{~mm}$, inner diameter $15 \mathrm{~mm}$ ) equipped with 18 thinner glass pipes (length $15 \mathrm{~mm}$, inner diameter $1.6 \mathrm{~mm}$ ) in its left half. The center of the unit is heated by passing an electric current through the nichrome wire surrounding it, while the ends are cooled by its copper support by heat conduction. The pump is set in a bell jar regulated at a low constant pressure. One of the ends of pump is open to the regulated circumstance in the bell jar, while the other is connected to a tank of volume $8 \times 10^{3} \mathrm{~cm}^{3}$ through a glass pipe (length $150 \mathrm{~mm}$, inner diameter $5 \mathrm{~mm}$ ) followed by a steel pipe (length $550 \mathrm{~mm}$, inner diameter $15 \mathrm{~mm}$ ). The experiment was performed from an almost uniform state throughout the system (the tank, pump, and regulated bell jar) by turning on an electric current through the nichrome wire. The temperature of the heated part increased from a room temperature $(\sim 290 \mathrm{~K})$ to around $450 \mathrm{~K}$, and the gas was evacuated from the tank by the temperature distribution established along the wall of pump. The experiment was performed for several values of the regulated pressure in the bell jar: 2, 5, 10, 20, 40, 60, 80, 120, 240Pa.

Numerical simulations of the experiment were performed by the use of the convection-diffusion system for the pump composed of circular pipes prepared in $[9,10]$. We treated the pumping system as 10 units cascade, thus ignoring the curved glass pipe connecting two subsystems. The numerical table of particle fluxes, which correspond to $M_{J}^{\alpha}$ in (4), is that based on the ellipsoidal statistical model for a single-component monatomic gas, though the experiment was done for the air. The reference pressure $p_{*}$ is the regulated pressure in the bell jar and temperature $T_{*}$ is $273 \mathrm{~K}$. We assume that the saw-tooth (and constant in time) distribution of wall temperature is instantaneously established after turning on the electric current, though it takes some time in the real experiment. The highest temperature is 440.8 , $440.8,440.8,440.8,447.4,456.6,463.2,472.4,486.8 \mathrm{~K}$ when $p_{*}=2,5,10,20,40,60,80,120,240 \mathrm{~Pa}$ respectively, while the lowest is commonly $293.4 \mathrm{~K}$. We ignored possible bends of glass and steel pipes connecting the pump to the tank and solved the equations inside them as if they were a part of the pump with uniform temperature distribution. At 


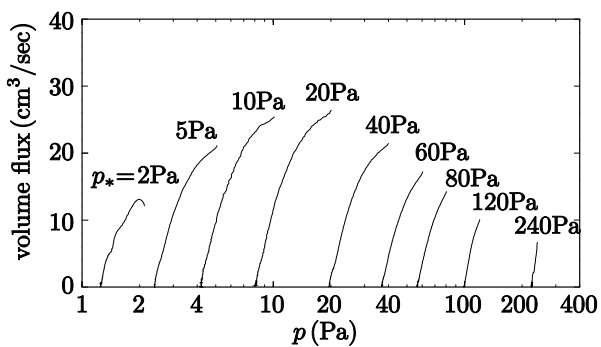

(a)

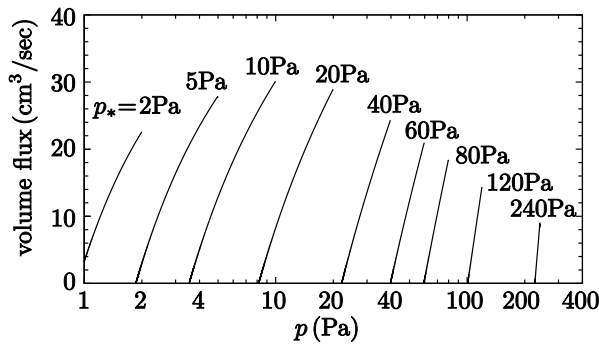

(b)

FIGURE 8. Evacuation speed versus the pressure in the tank during the evacuation process. (a) Experiment [6] (by courtesy of Dr. H. Sugimoto), (b) present numerical results.

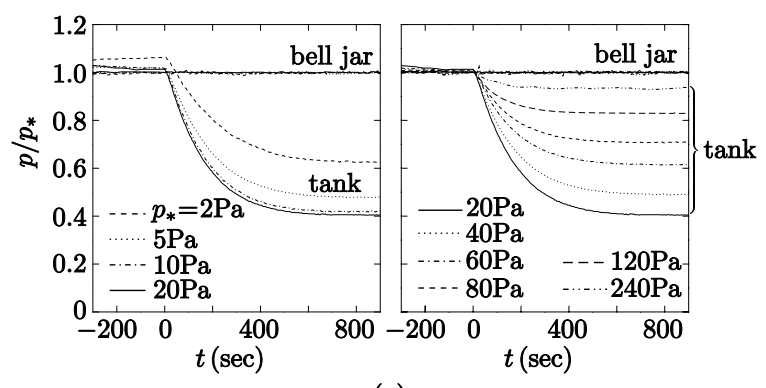

(a)

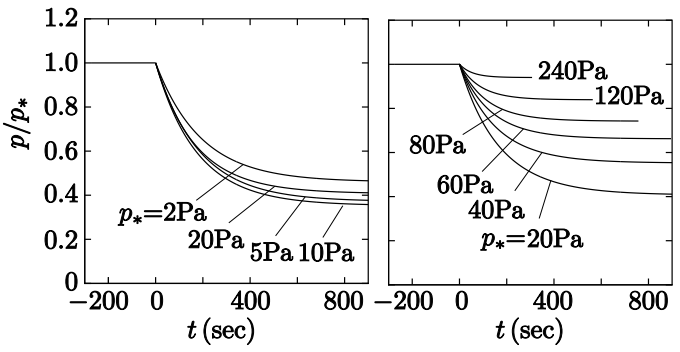

(b)

FIGURE 9. Time evolution of the pressure in the tank. (a) Experiment [6] (by courtesy of Dr. H. Sugimoto), (b) present numerical results.

the end that is open to the bell jar, the regulated pressure is imposed, while at the other end connected to the tank a (time-dependent) pressure is imposed as the boundary condition. During the process of time evolution, this pressure was evaluated from the tank volume and the evacuation speed (local in time) by assuming that the pressure inside the tank is uniform in space. The reference Knudsen number $\mathrm{K}_{*}$ was determined by the average molecular weight of the air (28.966) and the viscosity at the reference temperature $\left(1.71 \times 10^{-5} \mathrm{~Pa} \cdot \mathrm{s}\right)$. The mean free path of a molecule at 20 $\mathrm{Pa}$ is about $0.6 \mathrm{~mm}$.

The results of the experiment and simulation are shown in Figs. 8 and 9, respectively in panels (a) and (b). Figure 8 shows the volume flux (evacuation speed) as a function of the pressure $p$ in the tank for several $p_{*}$. The upper-right end point of each curve represents the initial evacuation speed and tank pressure. As time goes on, the representative point moves along each curve toward the lower-left end with zero volume flux, which is the final steady state. Figure 9 shows the time evolution of the pressure $p$ in the tank. As time goes on, the pressure in the tank decreases to reach the final steady state in at most 15 minutes.

In the present comparisons, the right half of elemental unit of the pump does not satisfy the narrow channel approximation and many simplifications have been made in modeling the experiment. Nevertheless, the simulation results well capture the features of experimental results, especially the consuming time quantitatively.

\section{REFERENCES}

1. M. Knudsen, Ann. Phys. (Leipzig) 31, 205-229 (1910).

2. G. Pham-Van-Diep, P. Keeley, E. P. Muntz, and D. P. Weaver, "A micromechanical Knudsen compressor," in Rarefied Gas Dynamics, Vol. I, edited by J. Harvey and G. Lord, Oxford University Press, London, 1995, pp. 715-721.

3. Y. Sone, Y. Waniguchi, and K. Aoki, Phys. Fluids 8, 2227-2235 (1996).

4. S. Takata, H. Sugimoto, and S. Kosuge, Eur. J. Mech. B-Fluids 26, 155-181 (2007).

5. K. Aoki and P. Degond, Multiscale Model. Simul. 1, 304-334 (2003).

6. Y. Sone and H. Sugimoto, "Vacuum Pump without a Moving Part and its Performance," in Rarefied Gas Dynamics, edited by A. D. Ketsdever and E. P. Muntz, American Institute of Physics, New York, 2003, pp. 1041-1048.

7. F. J. McCormack, Phys. Fluids 16, 2095-2105 (1973).

8. S. Kosuge and S. Takata, Eur. J. Mech. B-Fluids 27, 444-465 (2008).

9. K. Aoki, P. Degond, L. Mieussens, S. Takata, and H. Yoshida, Multiscale Model. Simul. 6, 1281-1316 (2008).

10. K. Aoki, S. Takata, and K. Kugimoto, "Diffusion approximation for the Knudsen compressor composed of circular tubes," in Rarefied Gas Dynamics, edited by T. Abe, American Institute of Physics, New York, 2009, pp. 953-958. 
Copyright of AIP Conference Proceedings is the property of American Institute of Physics and its content may not be copied or emailed to multiple sites or posted to a listserv without the copyright holder's express written permission. However, users may print, download, or email articles for individual use. 